\title{
Observation of Cellodextrin Accumulation Resulted from Non-Conventional Secretion of Intracellular $\beta$-Glucosidase by Engineered Saccharomyces cerevisiae Fermenting Cellobiose
}

\author{
Won-Heong Lee ${ }^{1,2 *}$ and Yong-Su Jin ${ }^{1}$ \\ 'Department of Food Science and Human Nutrition, and Carl R. Woese Institute for Genomic Biology, University of \\ Illinois at Urbana-Champaign, Urbana, IL 61801, USA \\ ${ }^{2}$ Department of Bioenergy Science and Technology, and Department of Integrative Food, Bioscience and \\ Biotechnology, Chonnam National University, Gwangju 61186, Republic of Korea
}

Although engineered Saccharomyces cerevisiae fermenting cellobiose is useful for the production of biofuels from cellulosic biomass, cellodextrin accumulation is one of the main problems reducing ethanol yield and productivity in cellobiose fermentation with S. cerevisiae expressing cellodextrin transporter (CDT) and intracellular $\beta$-glucosidase (GH1-1). In this study, we investigated the reason for the cellodextrin accumulation and how to alleviate its formation during cellobiose fermentation using engineered $S$. cerevisiae fermenting cellobiose. From the series of cellobiose fermentation using S. cerevisiae expressing only GH1-1 under several culture conditions, it was discovered that small amounts of GH1-1 were secreted and cellodextrin was generated through trans-glycosylation activity of the secreted GH1-1. As GH1-1 does not have a secretion signal peptide, non-conventional protein secretion might facilitate the secretion of GH1-1. In cellobiose fermentations with $S$. cerevisiae expressing only GH1-1, knockout of TLG2 gene involved in non-conventional protein secretion pathway significantly delayed cellodextrin formation by reducing the secretion of GH1-1 by more than $50 \%$. However, in cellobiose fermentations with S. cerevisiae expressing both GH1-1 and CDT-1, TLG2 knockout did not show a significant effect on cellodextrin formation, although secretion of GH1-1 was reduced by more than $40 \%$. These results suggest that the development of new intracellular $\beta$-glucosidase, not influenced by non-conventional protein secretion, is required for better cellobiose fermentation performances of engineered $S$. cerevisiae fermenting cellobiose.

Keywords: Cellulosic ethanol, engineered Saccharomyces cerevisiae, intracellular $\beta$-glucosidase, transglycosylation, non-conventional protein secretion

Received: May 14, 2021 Accepted: July 5, 2021

First published online: July 6, 2021

*Corresponding author Phone: +82-62-530-2046 Fax: +82-62-530-2047 E-mail:wonhlee@jnu.ac.kr

pISSN 1017-7825 elSSN 1738-8872

Copyright(C) 2021 by The Korean Society for Microbiology and Biotechnology

\section{Introduction}

Production of biofuels and chemicals from renewable biomass has received considerable attention as an alternative and sustainable way compared with the production of petroleum-based fuels and chemicals [1-3]. In particular, baker's yeast, Saccharomyces cerevisiae, has been employed as a microbial host to produce biofuels and chemicals from cellulosic biomass as this yeast has been utilized in ethanol production from starch-based biomass $[1,4,5]$. Because native $S$. cerevisiae cannot metabolize xylose, one of the abundant sugars (glucose and xylose) from enzymatic hydrolysis of cellulosic biomass, it has been engineered to metabolize xylose for efficient production of cellulosic ethanol [4-6]. Nevertheless, xylose metabolism by engineered S. cerevisiae is still limited by catabolite repression (glucose repression) in the presence of glucose [7-10], which has demanded new strategies for the efficient utilization of xylose by engineered S. cerevisiae without glucose repression.

Introduction of intracellular cellobiose assimilating pathway, composed of cellodextrin transporter (CDT-1 or CDT-2 from cellulolytic fungi, Neurospora crassa) and intracellular $\beta$-glucosidase (GH1-1 from $N$. crassa), into $S$. cerevisiae has been verified to be an effective approach for the production of cellulosic ethanol because it enables S. cerevisiae to ferment cellobiose and xylose simultaneously without glucose repression [11-13]. Furthermore, engineered $S$. cerevisiae fermenting cellobiose can enhance ethanol yield and productivity by co-fermentation of cellobiose and xylose compared with sequential fermentation of glucose and xylose [12, 13]. In addition, cellobiose-fermenting $S$. cerevisiae reduces enzyme costs and enhances ethanol productivity in simultaneous 
saccharification and fermentation (SSF) of cellulose $[14,15]$. However, engineered S. cerevisiae that utilizes intracellular cellobiose poses several problems in cellobiose fermentation despite of the benefits mentioned above. These problems include the accumulation of cellodextrin (e.g., cellotriose and cellotetraose) during cellobiose fermentation and slower cellobiose fermentation than glucose fermentation [12, 16-18]. In particular, cellodextrin accumulation increased when the cellobiose consumption rate by engineered S. cerevisiae was increased, which caused retardation of ethanol production during cellobiose fermentation [16-18].

Considering that cellodextrin is formed by trans-glycosylation of $\beta$-glucosidase when the concentration of cellobiose is extremely high [19-21], it would be conceivable that cellodextrin accumulation by engineered $S$. cerevisiae fermenting cellobiose might be caused by trans-glycosylation activities of intracellular and/or extracellular $\beta$-glucosidase. As such, we hypothesized that small amounts of GH1-1, even though GH1-1 lacks secretion signal-peptides, might be secreted (or leaked due to cell lysis) from the engineered S. cerevisiae cells by an unknown mechanism and secretion of GH1-1 might contribute to the accumulation of cellodextrin during cellobiose fermentation.

This study was undertaken to investigate the possibility for secretion of intracellular $\beta$-glucosidase by engineered S. cerevisiae utilizing intracellular cellobiose. To this end, we examined various culture conditions stimulating the secretion of GH1-1 by S. cerevisiae strain expressing only GH1-1 (without cellodextrin transporter). Moreover, we investigated the knockout effects of TLG2, which is involved in non-conventional protein secretion in S. cerevisiae, on the secretion of GH1-1 and cellodextrin accumulation by engineered S. cerevisiae strain fermenting cellobiose.

\section{Materials and Methods}

Strains and Plasmids

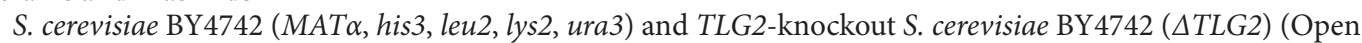
Biosystems, USA) were used as the host strains for expressing an intracellular $\beta$-glucosidase (GH1-1) with (or without) a cellodextrin transporter (CDT-1) from N. crassa. Plasmids pRS425-gh1-1 and pRS426-cdt1 were previously constructed for overexpression of intracellular $\beta$-glucosidase and cellodextrin transporter, respectively [11]. All strains and plasmids used in this study are listed in Table 1.

\section{Culture Conditions}

Synthetic complete (SC) medium (6.7 g/l yeast nitrogen base without amino acids and $0.625 \mathrm{~g} / \mathrm{l}$ complete supplement mixture without histidine, leucine and uracil) with appropriate nucleotide, amino acids and $20 \mathrm{~g} / \mathrm{l}$ glucose (dextrose) was used for seed cultivation. A single colony of each S. cerevisiae BY4742 strains from SCD agar plates was picked and inoculated into $5 \mathrm{ml}$ of SCD medium (20 g/l glucose, $\mathrm{pH}$ 6.5). Seed cultivation was performed in $10-\mathrm{mL}$ test tube at $30^{\circ} \mathrm{C}$ and $250 \mathrm{rpm}$.

For pre-cultivation of the engineered yeast strains, SCD medium ( $40 \mathrm{~g} / \mathrm{l}$ glucose, $\mathrm{pH} 6.5)$ was used. After $48 \mathrm{~h}$ of seed cultivation, cells were harvested and inoculated into $25 \mathrm{ml}$ of SCD medium. Pre-cultivation was performed in $125-\mathrm{mL}$ flask at $30^{\circ} \mathrm{C}$ and $250 \mathrm{rpm}$.

SC media at pH 6.5 (or pH 5.5) containing $20 \mathrm{~g} / \mathrm{l}$ cellobiose or YP media (10 g/l yeast extract and $20 \mathrm{~g} / \mathrm{l} \mathrm{Bacto-}$ peptone, pH 6.5 or 5.5) containing $20 \mathrm{~g} / \mathrm{l}$ cellobiose (or $80 \mathrm{~g} / \mathrm{l}$ cellobiose) were used for cellobiose fermentation of S. cerevisiae BY4742 strains expressing GH1-1 with or without CDT-1 [BY-B, BY-B ( $\Delta T L G 2)$, BY-BT, and BY-BT ( $\triangle T L G 2)$ strains]. YPD medium (20 g/l glucose, $\mathrm{pH}$ 6.5) was also used to compare glucose fermentations of BY-B and BY-B ( $\triangle T L G 2)$ strains with cellobiose fermentations. After $24 \mathrm{~h}$ of pre-cultivation, yeast cells were harvested. After washing three times with sterilized water, the cells were inoculated into $50 \mathrm{ml}$ of fermentation medium at initial optical density (OD) ${ }_{600}$ of $\sim 0.5$ (corresponds to $0.173 \mathrm{~g} / \mathrm{l}$ of dry cell mass). Cultivation was performed in 250 -ml flask at $30^{\circ} \mathrm{C}$ and $80 \mathrm{rpm}$ (micro-aerobic).

To determine the secretion of GH1-1 by the yeast strains, culture broth at the end of the fermentation was harvested and used to examine extracellular $\beta$-glucosidase activity.

\section{Analytical Methods}

Cell concentration was monitored by measuring OD at $600 \mathrm{~nm}$ using a UV-visible spectrophotometer (Biomate5, Thermo, USA). Glucose, cellobiose, cellodextrin and ethanol concentrations were determined by high

Table 1. The list of plasmids and S. cerevisiae strains used in this study.

\begin{tabular}{|c|c|c|}
\hline Plasmids and strains & Relevant features & Sources \\
\hline \multicolumn{3}{|l|}{ Plasmids } \\
\hline pRS425PGK & LEU2, $\mathrm{P}_{\mathrm{PGK}}-\mathrm{MCS}-\mathrm{T}_{\mathrm{CYC}}, 2 \mu$ origin, $\mathrm{Amp}^{\mathrm{r}}$ & {$[11]$} \\
\hline pRS426PGK & $U R A 3, \mathrm{P}_{\mathrm{PGK}}-\mathrm{MCS}-\mathrm{T}_{\mathrm{CYC}}, 2 \mu$ origin, $\mathrm{Amp}^{\mathrm{r}}$ & {$[11]$} \\
\hline pRS425-gh1-1 & LEU2, $\mathrm{P}_{\mathrm{PGK}^{-}}-g h 1-1-\mathrm{T}_{\mathrm{CYC}}, 2 \mu$ origin, $\mathrm{Amp}^{\mathrm{r}}$ & {$[11]$} \\
\hline pRS426-cdt1 & $U R A 3, \mathrm{P}_{\mathrm{PGK}}-c d t 1-\mathrm{T}_{\mathrm{CYC}}, 2 \mu$ origin, $\mathrm{Amp}^{\mathrm{r}}$ & {$[11]$} \\
\hline \multicolumn{3}{|c|}{ 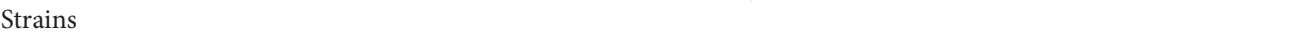 } \\
\hline BY4742 & MAT $\alpha$, his3, leu2, lys 2, ura3 & This study \\
\hline $\mathrm{BY} 4742(\triangle T L G 2)$ & BY4742, tlg2 $2:: \operatorname{KanMX}$ & This study \\
\hline BY-B & BY4742/pRS425-gh1-1/pRS426PGK & This study \\
\hline $\mathrm{BY}-\mathrm{B}(\triangle T L G 2)$ & BY4742 ( $\Delta T L G 2) / p R S 425-$ gh1-1/pRS426PGK & This study \\
\hline BY-BT & BY4742/pRS425-gh1-1/pRS426-cdt1 & This study \\
\hline $\mathrm{BY}-\mathrm{BT}(\triangle T L G 2)$ & BY4742 ( $\triangle T L G 2) / p R S 425-$ gh1-1/pRS426-cdt1 & This study \\
\hline
\end{tabular}


performance liquid chromatography (HPLC; Agilent Technologies 1200 Series, Agilent, USA) equipped with a refractive index (RI) detector using a Rezex ROA-Organic Acid $\mathrm{H}^{+}(8 \%)$ column (Phenomenex Inc., USA). The column was eluted with $0.005 \mathrm{~N}$ of $\mathrm{H}_{2} \mathrm{SO}_{4}$ at a flow rate of $0.6 \mathrm{ml} / \mathrm{min}$ at $50^{\circ} \mathrm{C}$.

Extracellular $\beta$-glucosidase activity was measured according to the methods reported previously [20-22]. The culture broth was harvested and centrifuged at $15,000 \times g$ for $20 \mathrm{~min}$ at $4^{\circ} \mathrm{C}$. After filtration of the supernatant with a $0.22-\mu \mathrm{m}$ spin filter, the filtrate $(500 \mu \mathrm{l})$ was mixed with the same volume of $50 \mathrm{mM}$ sodium citrate buffer $(\mathrm{pH} 4.8)$. After addition of $6.7 \mathrm{mM} p$-nitrophenyl- $\beta$-D-glucopyranoside ( $p$-NPG), release of $p$-nitrophenol $(p$-NP) was monitored by a spectrophotometer at $400 \mathrm{~nm}$. One unit (U) of $\beta$-glucosidase was defined as the amount of enzyme that catalyzes the release of $1 \mu \mathrm{mol}$ of $p$-NP per min at $30^{\circ} \mathrm{C}$. Volumetric extracellular $\beta$-glucosidase activity was expressed as units of $\beta$-glucosidase per liter. Specific extracellular $\beta$-glucosidase activity was expressed as units of $\beta$-glucosidase per gram of dry cell.

\section{Results and Discussion}

\section{Confirmation of GH1-1 Secretion from Engineered S. cerevisiae Expressing GH1-1}

Native S. cerevisiae strains, including BY4742, are unable to utilize cellobiose as they lack genes involved in the transport or degradation of cellobiose. Because there is no signal sequence for secretion of intracellular $\beta$-glucosidase (GH1-1) on the expression cassette of GH1-1 (pRS425-gh1-1) [11], GH1-1 must be located inside the cell when it is expressed in S. cerevisiae. Therefore, S. cerevisiae expressing only GH1-1 without cellobiose transporter (CDT1) cannot metabolize cellobiose and there is no possibility of cellobiose degradation and cellodextrin formation by the GH1-1 expressing $S$. cerevisiae because intracellular $\beta$-glucosidase cannot react with extracellular cellobiose.

To verify whether $S$. cerevisiae expressing only GH1-1 could consume cellobiose and whether cellodextrin formation could occur or not, the growth of the S. cerevisiae BY-B strain (BY4742 strain expressing GH1-1 only) on cellobiose was tested in a complex media (YP containing $20 \mathrm{~g} / \mathrm{l}$ cellobiose) at pH 6.5. Although BY-B could not transport cellobiose into the cell at all, cell growth, ethanol production, and accumulation of cellodextrin along with cellobiose consumption were observed in cellobiose fermentation with BY-B. As shown in Fig. 1A, $18.1 \mathrm{~g} / \mathrm{l}$ of cellobiose was consumed and $4.0 \mathrm{~g} / \mathrm{l}$ of ethanol was produced during cellobiose fermentation. Cellodextrin was accumulated up to $7.2 \mathrm{~g} / \mathrm{l}$ at $60 \mathrm{~h}$ and decreased to $3.4 \mathrm{~g} / \mathrm{l}$ at $72 \mathrm{~h}$ of fermentation. Considering that it is impossible
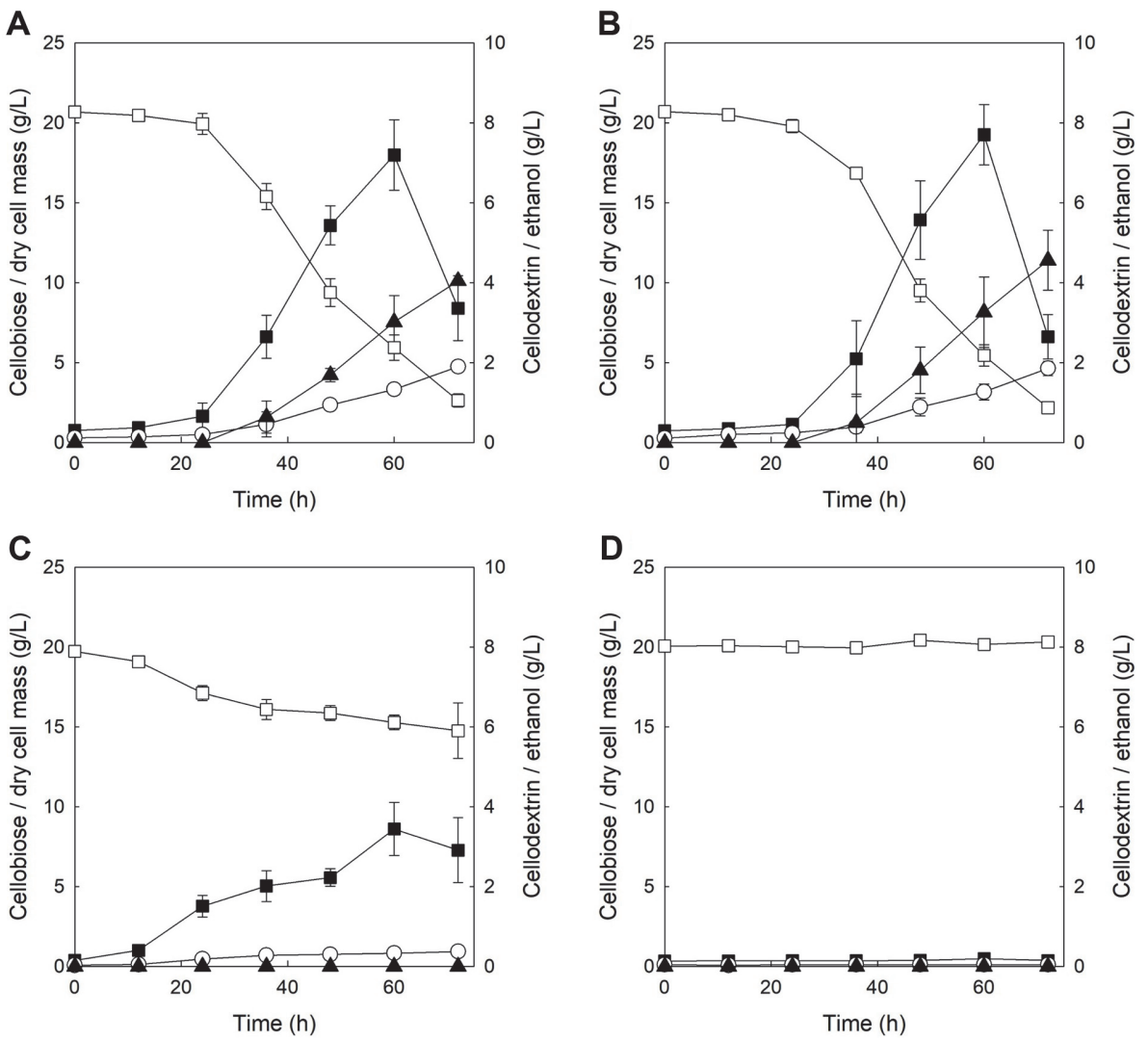

Fig. 1. Comparison of cellobiose fermentation profiles of $S$. cerevisiae expressing GH1-1 (BY-B) under different culture conditions. Complex (YP) medium with $20 \mathrm{~g} / \mathrm{l}$ cellobiose at $\mathrm{pH} 6.5$ (A), YP medium with $20 \mathrm{~g} / \mathrm{l}$ cellobiose at pH 5.5 (B), minimal (SC) medium with $20 \mathrm{~g} / 1$ cellobiose at pH 6.5 (C), and SC medium with $20 \mathrm{~g} / 1$ cellobiose at pH 5.5 (D). Symbols: cellobiose $(\square)$, dry cell mass $(\bigcirc)$, cellodextrin $(\boldsymbol{\square})$, and ethanol $(\boldsymbol{\Delta})$. All values of fermentations are mean values from two independent fermentation experiments, and error bars represent standard deviations. 
Table 2. Extracellular $\beta$-glucosidase accumulation by engineered S. cerevisiae expressing GH1-1 (BY-B) under different culture conditions.

\begin{tabular}{ccc}
\hline Culture conditions & $\begin{array}{c}\text { Volumetric extracellular } \beta \text {-glucosidase } \\
\text { activity in culture broth (U/L) }\end{array}$ & $\begin{array}{c}\text { Specific extracellular } \beta \text {-glucosidase } \\
\text { activity (U/g cell) }\end{array}$ \\
\hline YPC (20 g/l cellobiose, pH 6.5) & $14.9 \pm 3.80$ & $3.1 \pm 0.80$ \\
YPC (20 g/l cellobiose, pH 5.5) & $14.5 \pm 2.96$ & $3.1 \pm 0.64$ \\
SCC (20 g/l cellobiose, pH 6.5) & $0.6 \pm 0.32$ & $0.7 \pm 0.34$ \\
SCC (20 g/l cellobiose, pH 5.5) & 0.0 & 0.0 \\
\hline
\end{tabular}

The culture broth at the end $(72 \mathrm{~h})$ of the fermentation periods were used for the determination of extracellular $\beta$-glucosidase activity. All values are mean values from the samples of two independent fermentations, and error bars represent standard deviations.

for most sugars to pass through the yeast membrane by simple diffusion [23], we reasoned that formation and degradation of cellodextrin as well as degradation of cellobiose might occur at the outside of BY-B cells. Probably, BY-B may secrete a small amount of GH1-1 and the secreted GH1-1 might be involved in the degradation of cellobiose to glucose along with accumulation and degradation of cellodextrin. To validate whether small amounts of GH1-1 were secreted from the cell or not, the extracellular $\beta$-glucosidase activity of BY-B was measured using the culture broth at the end of the fermentation. As shown in Table 2, BY-B grown in YPC20 (pH 6.5) exhibited considerable level of extracellular $\beta$-glucosidase activity (14.9 U/L and 3.1 U/g cell). Considering that the average level of specific intracellular $\beta$-glucosidase activity of $S$. cerevisiae expressing GH1-1 grown in YPC was around $180 \mathrm{U} / \mathrm{g}$ cell (data not shown), it was estimated that approximately 2\% of GH1-1 expressed in the cell was secreted outside the cell. This observation is in good accordance to a previous report describing that frequency of trans-glycosylation reaction (cellodextrin formation) is dominant in the cellobiose hydrolysis reaction when cellobiose concentration is significantly higher than $\beta$-glucosidase concentration $[19,21]$. Results from cellobiose fermentation and extracellular $\beta$-glucosidase activity assay of BY-B clearly indicates that secretion of GH1-1 can contribute to extracellular cellobiose degradation and cellodextrin formation.

The growth of BY-B in YP containing $20 \mathrm{~g} / \mathrm{l}$ cellobiose under lower $\mathrm{pH}$ conditions was also tested to check if cellobiose consumption and cellodextrin formation by BY-B were affected by the change of external pH. Fig. 1B shows the cellobiose fermentation profile of BY-B in YPC20 at pH 5.5. It is interesting that almost similar profiles of cell growth, ethanol production, and cellodextrin accumulation were observed compared with those of cellobiose fermentation at $\mathrm{pH}$ 6.5. It was observed that $18.5 \mathrm{~g} / \mathrm{l}$ of cellobiose was consumed and $4.6 \mathrm{~g} / \mathrm{l}$ of ethanol was produced by BY-B from $20 \mathrm{~g} / \mathrm{l}$ of cellobiose. Cellodextrin was accumulated to $7.7 \mathrm{~g} / \mathrm{l}$ until $60 \mathrm{~h}$ and then rapidly decreased to $2.6 \mathrm{~g} / \mathrm{l}$. The transition from cellodextrin accumulation to cellodextrin degradation occurred when the cell concentrations were higher than $3 \mathrm{~g} / \mathrm{l}$ and the concentrations of cellobiose were lower than those of cellodextrin as shown in Figs. 1A and 1B. These results suggest that until $60 \mathrm{~h}$ of fermentation, sufficient amounts of $\beta$-glucosidase for degradation of cellodextrin (and cellobiose) might not be secreted by the engineered yeast. As trans-glycosylation and hydrolysis reaction are controlled by the concentrations of $\beta$-glucosidase and cellobiose, the transition from trans-glycosylation to hydrolysis reaction might have occurred around $60 \mathrm{~h}$ of the fermentation. BY-B grown in YPC20 ( $\mathrm{pH}$ 5.5) also exhibited almost the same level of extracellular $\beta$-glucosidase activity (14.5 U/L and 3.1 U/g cell) compared with that of cells grown in YPC20 at pH 6.5. Probably, activated protein synthesis in cells cultivated in a complex medium may lead to leakage of GH1-1 because YP medium provides many nutrients for protein synthesis, such as peptides, amino acids and nucleotides. As such, we reasoned that secretion of GH1-1 might be prevented (or reduced) if a minimal medium was used for cellobiose fermentation with BY-B. In addition, it could not be excluded that the putative cellobiose transporter in S. cerevisiae may transport cellobiose into the cell and then intracellular cellobiose was hydrolyzed to glucose or converted to cellodextrin by GH1-1.

To verify whether similar patterns of cellobiose utilization could be observed when the type of medium and external $\mathrm{pH}$ conditions were changed, cellobiose fermentation of BY-B was tested in SC containing $20 \mathrm{~g} / \mathrm{l}$ of cellobiose at different $\mathrm{pH}$ conditions (6.5 and 5.5). Fig. 1C shows the cellobiose fermentation profile of BY-B in SCC20 at pH 6.5. As expected, reduced cellobiose consumption was observed in the SCC medium. Although only $5.1 \mathrm{~g} / \mathrm{l}$ of cellobiose was consumed and ethanol was not produced during the fermentation, cellodextrin was accumulated at $3.4 \mathrm{~g} / \mathrm{l}$, which was around $70 \%$ conversion of the consumed cellobiose to cellodextrin. In additional cellobiose fermentation of BY-B which was extended until 144 hours in SCC20 at pH 6.5, after cellodextrin was accumulated up to $3.4 \mathrm{~g} / \mathrm{l}$ at $60 \mathrm{~h}$, cellobiose concentration slowly increased to $3.7 \mathrm{~g} / \mathrm{l}$ at $144 \mathrm{~h}$ of fermentation (data not shown). Compared with the results from cellobiose fermentation in YPC medium, less amount of GH1-1 seemed to be secreted from the cell due to reduced protein synthesis in SCC medium, which could explain the much lower cell concentration, no ethanol production and increased conversion ratio of cellobiose to cellodextrin. Fig. 1D shows the cellobiose fermentation profile of BY-B in SCC20 at pH 5.5. Interestingly, the BY-B strain exhibited neither cellobiose consumption nor cell growth. Moreover, cellodextrin accumulation was not observed. These results suggest that $S$. cerevisiae might not have any putative cellobiose transporter because the putative transporter (if it exists) might function at $\mathrm{pH} 5.5$ (optimum pH for S. cerevisiae growth) as well, even-though yeast cells cultured at pH 5.5 can show different cellobiose transport efficiency compared with those cultured at $\mathrm{pH}$ 6.5. These results also suggest that secretion of GH1-1 may be affected by growth conditions, such as external $\mathrm{pH}$ and supply of nutrients. To confirm that the level of GH1-1 secretion was affected by cell growth conditions, extracellular $\beta$-glucosidase activities of BY-B grown in SCC were measured. As 
Table 3. Summarized results from micro-aerobic fermentations of engineered S. cerevisiae strain expressing GH1-1 (BY-B) under different culture conditions.

\begin{tabular}{|c|c|c|c|c|}
\hline Culture conditions & $\begin{array}{c}\text { Final dry } \\
\text { cell mass }(\mathrm{g} / \mathrm{l})\end{array}$ & Final sugar $(\mathrm{g} / \mathrm{l})$ & $\begin{array}{l}\text { Maximum } \\
\text { ethanol }(\mathrm{g} / \mathrm{l})\end{array}$ & $\begin{array}{c}\text { Maximum } \\
\text { cellodextrin }(\mathrm{g} / \mathrm{l})\end{array}$ \\
\hline YPC (20 g/l cellobiose, $\mathrm{pH} 6.5)$ & $4.8 \pm 0.13$ & $2.6 \pm 0.41$ & $4.0 \pm 0.04$ & $7.2 \pm 0.88$ \\
\hline YPC ( $20 \mathrm{~g} / \mathrm{l}$ cellobiose, $\mathrm{pH} 5.5)$ & $4.6 \pm 0.45$ & $2.2 \pm 0.30$ & $4.6 \pm 0.75$ & $7.7 \pm 0.75$ \\
\hline SCC (20 g/l cellobiose, pH 6.5) & $0.9 \pm 0.11$ & $14.8 \pm 1.74$ & 0.0 & $3.4 \pm 0.66$ \\
\hline SCC (20 g/l cellobiose, $\mathrm{pH} 5.5)$ & $0.1 \pm 0.01$ & $20.3 \pm 0.05$ & 0.0 & 0.0 \\
\hline
\end{tabular}

Fermentation experiments were performed for $72 \mathrm{~h}$. All values are mean values from samples of two independent fermentations, and error bars represent standard deviations.

shown in Table 2, BY-B cells grown in SCC at pH 6.5 showed much lower level of extracellular $\beta$-glucosidase activity $(0.6 \mathrm{U} / \mathrm{L})$ than cells grown in YPC. In particular, BY-B cells grown at $\mathrm{pH} 5.5$ showed no extracellular $\beta$ glucosidase activity. These results indicate that secretion of GH1-1 might not occur in a minimal medium with lower $\mathrm{pH}$ conditions. In fact, secretion of GH1-1 from BY-B seemed to trigger cell growth, ethanol production and cellodextrin accumulation through extracellular cellobiose degradation and trans-glycosylation. The results from cellobiose fermentations of BY-B under different growth conditions are summarized in Table 3.

From previous studies on cellobiose fermentation with engineered S. cerevisiae strains expressing GH1-1 and cellodextrin transporters (CDT-1 or CDT-2 from N. crassa) [12, 16-18], it was hypothesized that cellodextrin accumulation may be primarily due to intracellular trans-glycosylation by GH1-1 through a series of steps, such as transportation of cellobiose, intracellular formation of cellodextrin, and export of cellodextrin by transporter (or diffusion). However, considering that small amounts of GH1-1 was secreted by BY-B in this study, it is also proposed that cellodextrin accumulation during cellobiose fermentation with engineered S. cerevisiae expressing GH1-1 and CDT may also be caused by secretion (or leakage) of GH1-1.

Effect of TLG2 Knockout on Cellobiose Fermentation with Engineered S. cerevisiae

As mentioned above, expression cassette for GH1-1 (pRS425-gh1-1) did not have any signal sequence for secretion, suggesting that GH1-1 may be secreted through a non-conventional protein secretion pathway [24-26], not a normal protein secretion pathway (endoplasmic reticulum- and Golgi-dependent protein secretion with Nterminal signal peptide) $[26,27]$ in S. cerevisiae. It has been reported that various proteins lacking signal peptide could be transported to the cell surface for their extracellular functions in yeast, such as S. cerevisiae and Candida albicans $[25,26]$. For example, signal peptide-less proteins in cytoplasm, such as glycolytic enzymes, chaperones and translation factors, have been frequently observed at the surface of yeast cells [24-26]. These studies proposed several potential mechanisms for non-conventional protein secretion; however, most mechanisms have not yet been clearly characterized [24-26]. Tlg2p is an endosomal specific t-SNARE protein that participates in fusion of endosome-derived vesicles, endocytosis, and exocytosis [24, 25, 28]. Moreover, Tlg2p is involved in nonconventional secretion of several metabolic enzymes in the cytoplasm [24, 25, 28, 29]. Previous studies have revealed that TLG2 knockout significantly reduces non-conventional secretion of Acb1p (acyl-CoA-binding protein in fatty acid biosynthesis) and Enolp (enolase in glycolysis) in S. cerevisiae [28, 29].

Consequently, cellobiose fermentations with two S. cerevisiae strains expressing only GH1-1, BY-B and TLG2knockout BY-B, were performed to validate whether deletion of TLG2 gene could affect secretion of GH1-1 and accumulation of cellodextrin. Figs. $2 \mathrm{~A}$ and $2 \mathrm{~B}$ compare the cellobiose fermentation profiles between BY-B and $\mathrm{BY}-\mathrm{B}(\triangle T L G 2)$ in YPC ( $20 \mathrm{~g} / \mathrm{l}$ cellobiose, $\mathrm{pH}$ 6.5). Although BY-B showed almost the same fermentation profiles as previous fermentation under the same conditions (Fig. 2A), BY-B ( $\triangle T L G 2)$ showed considerably slower cell growth and cellobiose consumption with reduced ethanol production and decreased cellodextrin accumulation than BY-B (Fig. 2B). In cellobiose fermentation with BY-B ( $\triangle T L G 2), 2.3 \mathrm{~g} / \mathrm{l}$ of maximum ethanol production and $3.9 \mathrm{~g} / \mathrm{l}$ of maximum cellodextrin accumulation were observed, which corresponded to $45 \%$ and $37 \%$ reduction, respectively, compared with those observed in cellobiose fermentation with BY-B. Probably, TLG2 knockout may hinder the non-conventional secretion of GH1-1, which could lead to delayed cellobiose metabolism in BY-B $(\triangle T L G 2)$. As presented in Table 4, extracellular $\beta$-glucosidase activity assay performed on culture broth at the end of fermentations showed that secretion of GH1-1 from BY-B ( $\triangle T L G 2)$ decreased by $56 \%$ than that from BY-B [10.8 U/L in BY-B ( $\triangle T L G 2)$ vs. $24.5 \mathrm{U} / \mathrm{L}$ in BY-B], suggesting that TLG2 knockout may prevent extracellular cellodextrin formation. However, it could not be excluded that TLG2 knockout may inhibit cell growth, irrespective of preventing GH1-1 secretion.

To determine whether TLG2 knockout could inhibit cell growth even under normal condition, glucose fermentations with BY-B and BY-B $(\triangle T L G 2)$ were performed in YPD $(20 \mathrm{~g} / \mathrm{l}$ glucose, $\mathrm{pH}$ 6.5). As illustrated in Figs. $2 \mathrm{C}$ and $2 \mathrm{D}$, the two strains showed almost the same fermentation profiles, including glucose consumption, cell growth and ethanol production, indicating that TLG2 knockout did not affect cell growth and sugar metabolism at all. Interestingly, secretion of GH1-1 rarely occurred in both strains cultured in glucose [0.3 U/L in BY-B; 0.1 U/L in BY-B ( $\triangle T L G 2)]$ as shown in Table 4, which suggests that secretion of GH1-1 may be induced when there was a specific selective pressure, such as utilizing cellobiose as the sole carbon source, on cell growth. The results from cellobiose (or glucose) fermentations with BY-B and BY-B ( $\triangle T L G 2$ ) are summarized in Table 5.

To elucidate whether similar patterns of cellobiose fermentation profiles could be observed when the concentration of cellobiose was increased, cellobiose fermentations with two strains were performed in YP 

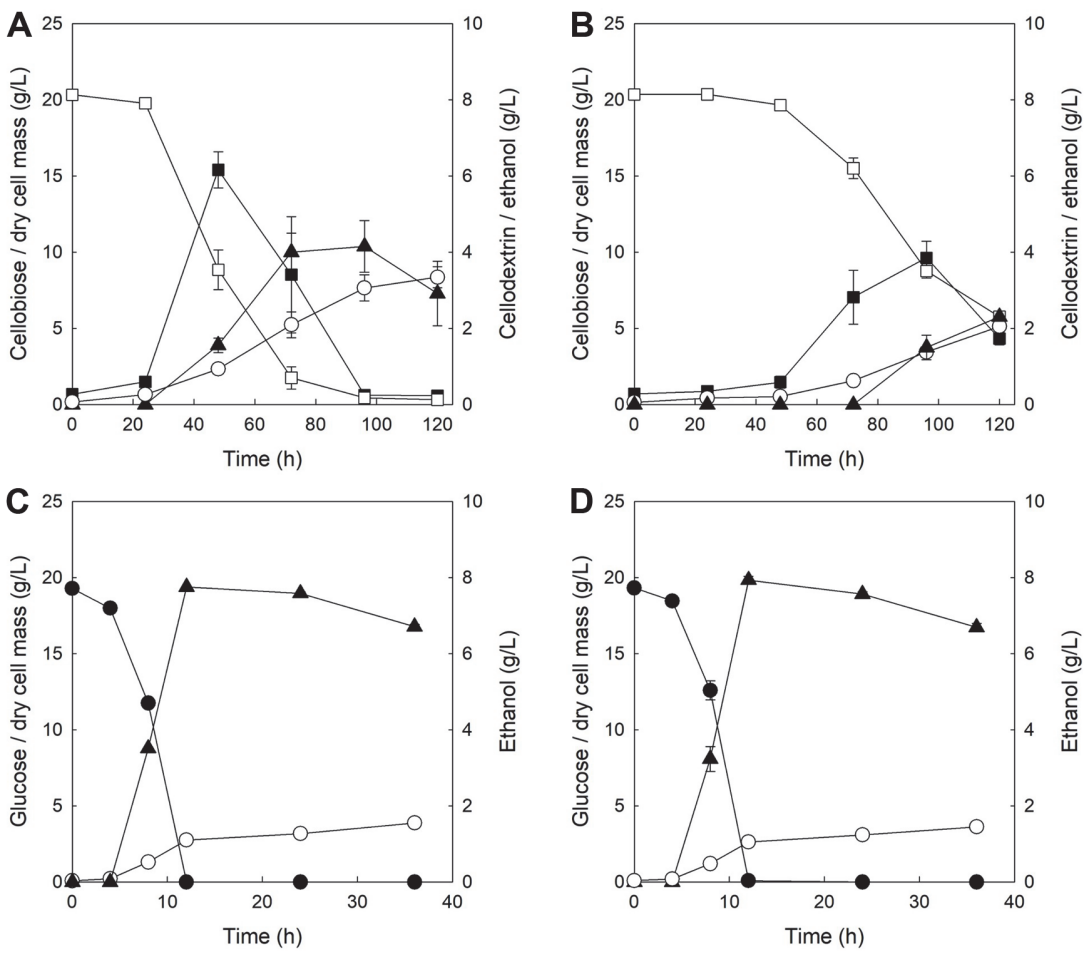

Fig. 2. Comparison of fermentation profiles of S. cerevisiae strains expressing GH1-1 [BY-B and BY-B ( $\Delta T L G 2)]$ under different culture conditions. BY-B in YP medium with $20 \mathrm{~g} / \mathrm{l}$ cellobiose at $\mathrm{pH} 6.5(\mathrm{~A}), \mathrm{BY}-\mathrm{B}(\triangle T L G 2)$ in YP medium with $20 \mathrm{~g} / \mathrm{l}$ cellobiose at $\mathrm{pH} 6.5$ (B), BY-B in YP medium with $20 \mathrm{~g} / \mathrm{l}$ glucose at $\mathrm{pH} 6.5(\mathrm{C})$, and BY-B $(\Delta T L G 2)$ in YP medium with $20 \mathrm{~g} / \mathrm{l}$ glucose at pH $6.5(\mathbf{D})$. Symbols: cellobiose $(\square)$, glucose $(\mathbf{O})$, dry cell mass $(\bigcirc)$, cellodextrin $(\boldsymbol{\square})$, and ethanol ( $\mathbf{\Delta})$. All values of fermentations are mean values from two independent fermentations, and error bars represent standard deviations.

Table 4. Extracellular $\beta$-glucosidase accumulation by engineered $S$. cerevisiae strains in YP medium with cellobiose (or glucose) at pH 6.5.

\begin{tabular}{clcc}
\hline $\begin{array}{c}\text { Culture } \\
\text { conditions }\end{array}$ & \multicolumn{1}{c}{ Strains } & $\begin{array}{c}\text { Volumetric extracellular } \beta \text {-glucosidase } \\
\text { activity in culture broth }(\mathrm{U} / \mathrm{L})\end{array}$ & $\begin{array}{c}\text { Specific extracellular } \beta \text {-glucosidase } \\
\text { activity }(\mathrm{U} / \mathrm{g} \text { cell })\end{array}$ \\
\hline YPC20 & BY-B & $24.5 \pm 2.76$ & $2.9 \pm 0.33$ \\
& BY-B $(\triangle T L G 2)$ & $10.8 \pm 0.90$ & $2.1 \pm 0.17$ \\
YPD20 & BY-B & $0.3 \pm 0.15$ & $0.1 \pm 0.04$ \\
& BY-B $(\triangle T L G 2)$ & $0.1 \pm 0.11$ & $0.0 \pm 0.03$ \\
YPC75 & BY-B & $30.1 \pm 2.61$ & $3.1 \pm 0.27$ \\
& BY-B $(\triangle T L G 2)$ & $13.0 \pm 1.80$ & $2.1 \pm 0.29$ \\
& BY-BT & $38.6 \pm 7.41$ & $4.0 \pm 0.77$ \\
& BY-BT $(\triangle T L G 2)$ & $21.8 \pm 2.39$ & $2.6 \pm 0.29$ \\
\hline
\end{tabular}

The culture broth at the end (120, 36, and $144 \mathrm{~h}$ for YPC20, YPD20, and YPC75, respectively) of the fermentation periods were used for determination of extracellular $\beta$-glucosidase activity. All values are mean values from samples of two independent fermentations, and error bars represent standard deviations.

Table 5. Summarized results from micro-aerobic fermentations of engineered S. cerevisiae strains in YP medium with cellobiose (or glucose) at pH 6.5.

\begin{tabular}{|c|c|c|c|c|c|}
\hline $\begin{array}{l}\text { Culture } \\
\text { conditions }\end{array}$ & Strains & $\begin{array}{c}\text { Final dry } \\
\text { cell mass }(\mathrm{g} / \mathrm{l})\end{array}$ & Final sugar (g/l) & $\begin{array}{l}\text { Maximum } \\
\text { ethanol }(\mathrm{g} / \mathrm{l})\end{array}$ & $\begin{array}{c}\text { Maximum } \\
\text { cellodextrin }(\mathrm{g} / \mathrm{l})\end{array}$ \\
\hline \multirow{2}{*}{ YPC20 } & BY-B & $8.4 \pm 0.69$ & $0.3 \pm 0.03$ & $4.2 \pm 0.68$ & $6.2 \pm 0.48$ \\
\hline & BY-B $(\triangle T L G 2)$ & $5.1 \pm 0.07$ & $5.8 \pm 0.08$ & $2.3 \pm 0.03$ & $3.9 \pm 0.43$ \\
\hline \multirow[t]{2}{*}{ YPD20 } & BY-B & $3.9 \pm 0.14$ & 0.0 & $7.8 \pm 0.01$ & 0.0 \\
\hline & $\mathrm{BY}-\mathrm{B}(\triangle T L G 2)$ & $3.6 \pm 0.21$ & 0.0 & $7.9 \pm 0.10$ & 0.0 \\
\hline \multirow[t]{4}{*}{ YPC75 } & BY-B & $9.7 \pm 0.17$ & $8.1 \pm 0.58$ & $13.5 \pm 0.46$ & $28.0 \pm 0.68$ \\
\hline & BY-B $(\triangle T L G 2)$ & $6.3 \pm 0.06$ & $21.3 \pm 0.03$ & $7.6 \pm 0.06$ & $23.7 \pm 0.56$ \\
\hline & BY-BT & $9.6 \pm 0.09$ & $0.9 \pm 0.05$ & $25.2 \pm 0.33$ & $25.0 \pm 0.26$ \\
\hline & BY-BT $(\triangle T L G 2)$ & $8.3 \pm 0.69$ & $1.0 \pm 0.28$ & $22.8 \pm 0.72$ & $21.7 \pm 1.19$ \\
\hline
\end{tabular}

Fermentation experiments with YPC20, YPD20, and YPC75 were performed for 120, 36, and $144 \mathrm{~h}$, respectively. 

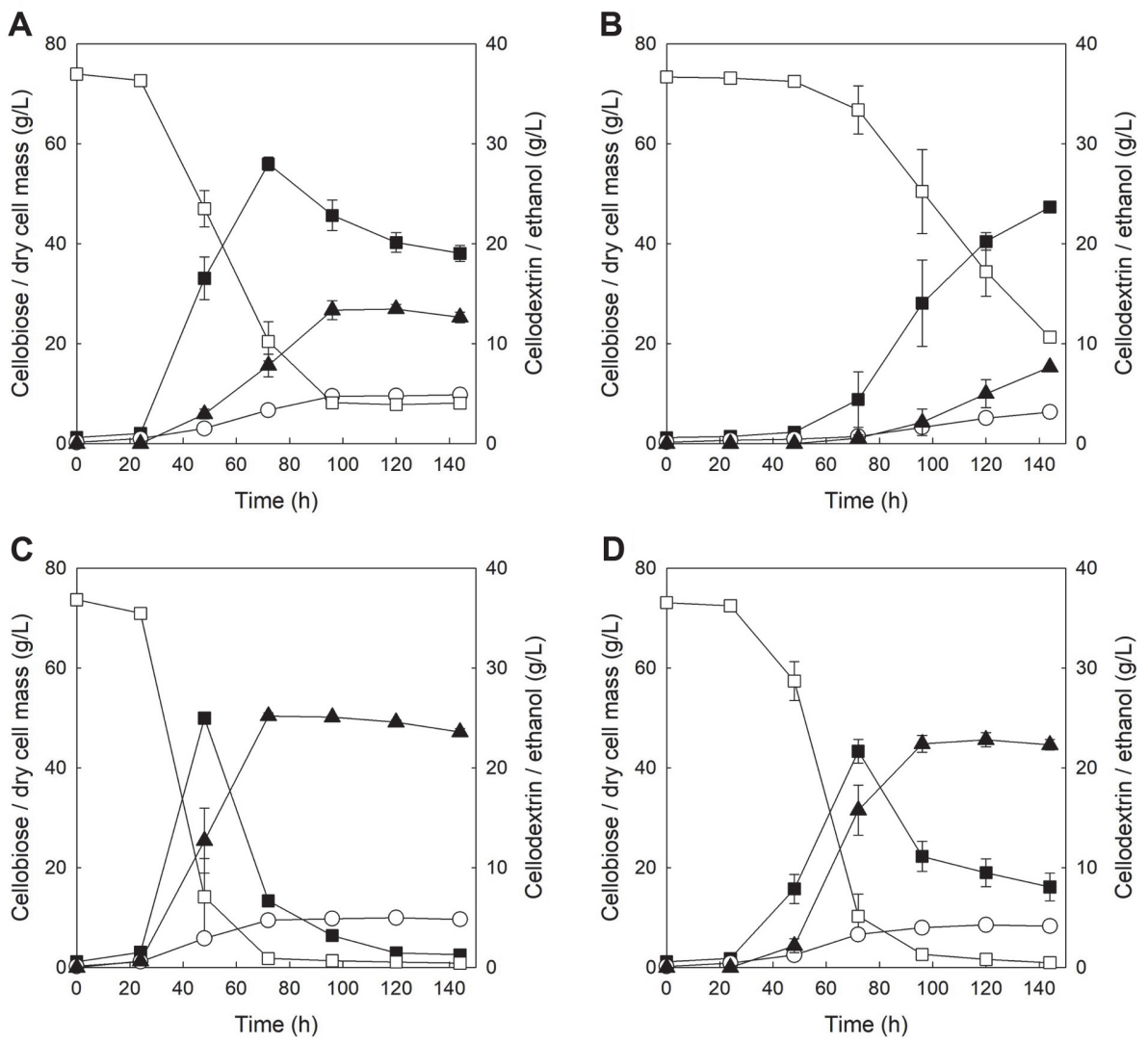

Fig. 3. Comparison of fermentation profiles of $S$. cerevisiae strains expressing only GH1-1 [BY-B and BY-B $(\triangle T L G 2)]$ and S. cerevisiae strains expressing both GH1-1 with CDT-1 [BY-BT and BY-BT ( $\triangle T L G 2)]$ in YP medium with 75 g/l cellobiose at pH 6.5. BY-B (A), BY-B ( $\triangle T L G 2)($ B), BY-BT (C), and BY-BT ( $\triangle T L G 2)($ D). Symbols: cellobiose $(\square)$, dry cell mass $(\bigcirc)$, cellodextrin $(\boldsymbol{\square})$, and ethanol $(\boldsymbol{\Delta})$. All values of fermentations are mean values from two independent fermentations, and error bars represent standard deviations.

containing $75 \mathrm{~g} / \mathrm{l}$ cellobiose at $\mathrm{pH}$ 6.5. As shown in Figs. 3A and 3B, cellobiose fermentation profiles of BY-B and $\mathrm{BY}-\mathrm{B}(\triangle T L G 2)$ under high cellobiose condition were similar to those under low cellobiose condition, respectively. During cellobiose fermentation with BY-B as shown in Fig. 3A, $65.9 \mathrm{~g} / \mathrm{l}$ of cellobiose was consumed and $13.5 \mathrm{~g} / \mathrm{l}$ of ethanol was produced along with accumulation and re-assimilation of cellodextrin from $28.0 \mathrm{~g} / \mathrm{l}$ to $19.0 \mathrm{~g} / \mathrm{l}$. It is interesting to note that $8.1 \mathrm{~g} / \mathrm{l}$ of cellobiose and $19.0 \mathrm{~g} / \mathrm{l}$ of cellodextrin remained as residual sugars until the end of the fermentation, which was different from the result observed in fermentation with low concentration of cellobiose. Probably, the amount of GH1-1 secreted from BY-B is thought to be insufficient to degrade all of the cellobiose existing at high concentration. Furthermore, the total amount of GH1-1 that a single cell could secrete is thought to be maintained at a constant level, regardless of the initial cellobiose concentration. As shown in Table 4, specific extracellular $\beta$-glucosidase activity of BY-B grown in YPC75 was similar to BY-B grown in YPC20 (3.1 U/g cell in YPC75 vs. $2.9 \mathrm{U} / \mathrm{g}$ cell in YPC20 and $3.1 \mathrm{U} / \mathrm{g}$ cell in previous YPC20). Meanwhile, during cellobiose fermentation with BY-B $(\triangle T L G 2)$ as shown in Fig. 3B, $52.1 \mathrm{~g} / \mathrm{l}$ of cellobiose was consumed for production of $7.6 \mathrm{~g} / \mathrm{l}$ ethanol and accumulation of $23.7 \mathrm{~g} / \mathrm{l}$ cellodextrin, which decreased by $21 \%, 45 \%$, and $15 \%$, respectively, compared with cellobiose fermentation with BY-B. As mentioned above, it was supposed that TLG2 knockout may limit secretion of GH1-1 and result in delayed cellobiose fermentation (with delayed cellodextrin formation). Table 4 indicates that extracellular secretion of GH1-1 from BY-B ( $\triangle T L G 2)$ grown in YPC75 was reduced by $57 \%$ than that from BY-B [13.0 U/L in BY-B $(\triangle T L G 2)$ vs. $30.1 \mathrm{U} / \mathrm{L}$ in BY-B], which is similar to the result from fermentation in YPC20.

Because TLG2 knockout was effective in preventing GH-1 secretion in cellobiose fermentation with BY-B ( $\triangle T L G 2$ ), fermentations of $75 \mathrm{~g} / \mathrm{l}$ cellobiose with BY-BT and BY-BT ( $\triangle T L G 2)$ were also tested to check whether TLG2 knockout could affect cellodextrin accumulation and ethanol production in cellobiose fermentation with yeast strain expressing GH1-1 and CDT-1 together. As confirmed in Figs. 2B and 2D, the deletion of TLG2 did not cause any phenotypic changes under YPD conditions but the deletion of TLG2 prevented cellodextrin accumulation of BY-B ( $\triangle T L G 2)$ strain under YPC conditions. Taken together, we expected that TLG2 deletion might affect cellodextrin accumulation and overall fermentation performances in YPC fermentation by BY-BT $(\triangle T L G 2)$.

In cellobiose fermentation with BY-BT in YPC75 ( $\mathrm{pH}$ 6.5) as shown in Fig. 3C, most of the initial cellobiose was 
consumed and $25.2 \mathrm{~g} / \mathrm{l}$ of ethanol was produced at $72 \mathrm{~h}$ of the fermentation. Cellodextrin was accumulated to $25.0 \mathrm{~g} / \mathrm{l}$ at $48 \mathrm{~h}$ and rapidly decreased until $72 \mathrm{~h}$ of fermentation, after which it decreased slowly. In particular, during the period for slow consumption of cellodextrin ( $72 \mathrm{~h}$ to $144 \mathrm{~h}$ ), no more ethanol was produced, which may be the reason that cellodextrin accumulation reduced ethanol yield and productivity in cellobiose fermentation. In addition, secretion of GH1-1 from BY-BT was observed (38.6 U/L and 4.0 U/g cell) as shown in Table 4, which supports the result that cellodextrin accumulation by S. cerevisiae expressing GH1-1 and CDT could be due to the secretion of GH1-1. Fig. 3D shows cellobiose fermentation profiles of BY-BT ( $\triangle T L G 2)$ in YPC75 ( $\mathrm{pH} 6.5$ ). Unfortunately, considerably reduced cellobiose consumption, less production of ethanol, and slightly reduced cellodextrin accumulation were observed in BY-BT $(\triangle T L G 2)$, although secretion of GH1-1 decreased by more than $40 \%$ compared with BY-BT [21.8 U/L in BY-BT ( $\triangle T L G 2)$ vs. $38.6 \mathrm{U} / \mathrm{L}$ in BY-BT] as shown in Table 4. TLG2 knockout led to retardation of cellodextrin formation; however, cellobiose consumption and ethanol production were also delayed. In particular, the accumulated cellodextrin was re-assimilated considerably slowly, and $8.1 \mathrm{~g} / \mathrm{l}$ of cellodextrin remained until the end of the fermentation. These results suggest that TLG2 knockout may affect the function (or expression or delivery) of CDT-1 because rapid consumption of cellodextrin as well as cellobiose was observed in the case of cellobiose fermentation with BY-BT. Consequently, TLG2 knockout is assumed to have negative effects on cellobiose metabolism in S. cerevisiae expressing GH1-1 and CDT-1, although it could prevent non-conventional secretion of GH1-1. The results from cellobiose fermentations with four strains [BY-B, BY-B ( $\triangle T L G 2), \mathrm{BY}-\mathrm{BY}$, and BY-B $(\triangle T L G 2)]$ are summarized in Table 5.

Cellodextrin accumulation by $S$. cerevisiae expressing GH1-1 and CDT has been regarded as one of the main problems decreasing fermentation performance in terms of ethanol yield and productivity in the cellobiose fermentation [16-18]. In this study, it was observed that secretion of a small amount of GH1-1 could cause extracellular formation of cellodextrin. Furthermore, it has been observed that knockout of TLG2, involved in non-conventional protein secretion, was effective in reducing the secretion of GH1-1 lacking signal peptide for classical protein secretion, resulting in delayed and reduced cellodextrin accumulation in cellobiose fermentation with $S$. cerevisiae expressing only GH1-1. However, TLG2 knockout negatively influenced on cellodextrin accumulation in cellobiose fermentation with $S$. cerevisiae expressing both GH1-1 and CDT-1, resulting in delayed cellobiose consumption and no significant change in cellodextrin accumulation. Perhaps, other genes involved in non-conventional protein secretion $[24,26]$ need to be investigated for prevention of GH1-1 secretion and reduction of cellodextrin formation in S. cerevisiae expressing GH1-1 and CDT-1. Probably, additional knockout of other genes along with TLG2 might be effective on cellodextrin accumulation in S. cerevisiae fermenting cellobiose. In addition, unknown properties of heterologous GH1-1 may stimulate its secretion from S. cerevisiae because GH1-1 was originated from fungi [11], which suggests that other intracellular $\beta$-glucosidase genes from other yeast species (e.g., $B G L$ genes from Pichia stipitis) [30] need to be tested to confirm whether they are also secreted through non-conventional protein secretion in S. cerevisiae.

\section{Acknowledgments}

This research was supported by the National Research Foundation of Korea (NRF) grant funded by the Korea government (MSIT) (No. NRF-2019R1F1A1059516). This work was also supported by Korea Institute of Planning and Evaluation for Technology in Food, Agriculture and Forestry (IPET) and Korea Smart Farm R\&D Foundation (KosFarm) through Smart Farm Innovation Technology Development Program, funded by Ministry of Agriculture, Food and Rural Affairs (MAFRA) and Ministry of Science and ICT (MSIT), Rural Development Administration (RDA) (No. 421045-03).

\section{Conflict of interest}

The authors have no financial conflicts of interest to declare.

\section{References}

1. Liu CG, Xiao Y, Xia XX, Zhao XQ, Peng L, Srinophakun P, et al. 2019. Cellulosic ethanol production: progress, challenges and strategies for solutions. Biotechnol. Adv. 37: 491-504.

2. Lynd LR. 2017. The grand challenge of cellulosic biofuels. Nat. Biotechnol. 35: 912-915.

3. Lynd LR, Liang X, Biddy MJ, Allee A, Cai H, Foust T, et al. 2017. Cellulosic ethanol: status and innovation. Curr. Opin. Biotechnol. 45: 202-211.

4. Jin YS, Cate JH. 2017. Metabolic engineering of yeast for lignocellulosic biofuel production. Curr. Opin. Chem. Biol. 41: 99-106.

5. Turner TL, Kim H, Kong II, Liu JJ, Zhang GC, Jin YS. 2016. Engineering and evolution of Saccharomyces cerevisiae to produce biofuels and chemicals. Adv. Biochem. Eng. Biotechnol. 162:175-215.

6. Kim SR, Park YC, Jin YS, Seo JH. 2013. Strain engineering of Saccharomyces cerevisiae for enhanced xylose metabolism. Biotechnol. Adv. 31: 851-861.

7. Farwick A, Bruder S, Schadeweg V, Oreb M, Boles E. 2014. Engineering of yeast hexose transporters to transport D-xylose without inhibition by D-glucose. Proc. Natl. Acad. Sci. USA 111: 5159-5164.

8. Gancedo JM. 1998. Yeast carbon catabolite repression. Microbiol. Mol. Biol. Rev. 62: 334-361.

9. Hou J, Qiu C, Shen Y, Li H, Bao X. 2017. Engineering of Saccharomyces cerevisiae for the efficient co-utilization of glucose and xylose. FEMS. Yeast. Res. 17: 1-11.

10. Subtil T, Boles E. 2012. Competition between pentoses and glucose during uptake and catabolism in recombinant Saccharomyces cerevisiae. Biotechnol. Biofuels 5: 1-12.

11. Galazka JM, Tian C, Beeson WT, Martinez B, Glass NL, Cate JH. 2010. Cellodextrin transport in yeast for improved biofuel production. Science 330: 84-86

12. Ha SJ, Galazka JM, Kim SR, Choi JH, Yang X, Seo JH, et al. 2011. Engineered Saccharomyces cerevisiae capable of simultaneous cellobiose and xylose fermentation. Proc. Natl. Acad. Sci. USA 108: 504-509. 
13. Kim SR, Ha SJ, Wei N, Oh EJ, Jin YS. 2012. Simultaneous co-fermentation of mixed sugars: a promising strategy for producing cellulosic ethanol. Trends Biotechnol. 30: 274-282.

14. Lee WH, Jin YS. 2017. Improved ethanol production by engineered Saccharomyces cerevisiae expressing a mutated cellobiose transporter during simultaneous saccharification and fermentation. J. Biotechnol. 245: 1-8.

15. Lee WH, Nan H, Kim HJ, Jin YS. 2013. Simultaneous saccharification and fermentation by engineered Saccharomyces cerevisiae without supplementing extracellular $\beta$-glucosidase. J. Biotechnol. 167: 316-322.

16. Bae YH, Kang KH, Jin YS, Seo JH. 2014. Molecular cloning and expression of fungal cellobiose transporters and $\beta$-glucosidases conferring efficient cellobiose fermentation in Saccharomyces cerevisiae. J. Biotechnol. 169: 34-41.

17. Kim H, Lee WH, Galazka JM, Cate JH, Jin YS. 2014. Analysis of cellodextrin transporters from Neurospora crassa in Saccharomyces cerevisiae for cellobiose fermentation. Appl. Microbiol. Biotechnol. 98: 1087-1094.

18. Kim HJ, Lee WH, Turner TL, Kwak S, Jin YS. 2019. An extra copy of the $\beta$-glucosidase gene improved the cellobiose fermentation capability of an engineered Saccharomyces cerevisiae strain. 3 Biotech. 9: 1-10.

19. Bohlin C, Praestgaard E, Baumann MJ, Borch K, Praestgaard J, Monrad RN, et al. 2013. A comparative study of hydrolysis and transglycosylation activities of fungal $\beta$-glucosidases. Appl. Microbiol. Biotechnol. 97: 159-169.

20. Teugjas H, Väljamäe P. 2013. Selecting $\beta$-glucosidases to support cellulases in cellulose saccharification. Biotechnol. Biofuels 6: 1-13.

21. Zhao J, Shi D, Yang S, Lin H, Chen H. 2020. Identification of an intracellular $\beta$-glucosidase in Aspergillus niger with transglycosylation activity. Appl. Microbiol. Biotechnol. 104: 8367-8380.

22. Njokweni AP, Rose SH, van Zyl WH. 2012. Fungal $\beta$-glucosidase expression in Saccharomyces cerevisiae. J. Ind. Microbiol. Biotechnol. 39: $1445-1452$

23. Lagunas R. 1993. Sugar transport in Saccharomyces cerevisiae. FEMS. Microbiol. Rev. 10: 229-242.

24. Malhotra V. 2013. Unconventional protein secretion: an evolving mechanism. EMBO. J. 32: 1660-1664.

25. Miura N, Ueda M. 2018. Evaluation of unconventional protein secretion by Saccharomyces cerevisiae and other fungi. Cells 7: 128 .

26. Nombela C, Gil C, Chaffin WL. 2006. Non-conventional protein secretion in yeast. Trends. Microbiol. 14: 15-21.

27. Delic M, Valli M, Graf AB, Pfeffer M, Mattanovich D, Gasser B. 2013. The secretory pathway: exploring yeast diversity. FEMS. Microbiol. Rev. 37: 872-914.

28. Miura N, Kirino A, Endo S, Morisaka H, Kuroda K, Takagi M, Ueda M. 2012. Tracing putative trafficking of the glycolytic enzyme enolase via SNARE-driven unconventional secretion. Eukaryot. Cell 11: 1075-1082.

29. Duran JM, Anjard C, Stefan C, Loomis WF, Malhotra V. 2010. Unconventional secretion of Acb1 is mediated by autophagosomes. J. Cell. Biol. 188: 527-536.

30. Jeffries TW, Van Vleet JR. 2009. Pichia stipitis genomics, transcriptomics, and gene clusters. FEMS Yeast Res. 9: 793-807. 